\title{
The Segmentation of Orchid Leaf Disease Spot Based On Lab Color Space and Otsu
}

\author{
Danni Ci \\ Tianjin Key Laboratory of Information Sensing and Intelligent Control, Tianjin University of Technology and Education No.1310,Dagu \\ South Road, Hexi District, Tianjin 300222, China
}

\begin{abstract}
Due to the unreasonable fertilizer or some other combination of unpredictable factors, makes the flowers in the greenhouse to the disease, directly affect the production of our country the output of the flower. In order to avoid this kind of diseases affect other flowers in the greenhouse, need to be recognition and diagnosis, the priority is split and extract the disease spot. We Put forward a method that the Lab space is used to segment the image of the disease leaf of the orchid by adopting the Otsu the blade was divided, complete separation of normal area and disease area of the leaf, finally we restore the color images of disease spot. The test results show that the proposed method can accurately extract leaf disease spot area.
\end{abstract}

Keywords: Otsu, Lab space, disease spot area

\section{Introduction}

In recent years, the flower has become almost essential items for every household. The cultivation of flowers is generally carried out in the greenhouse which is facilities for the cultivation of crops by controlling the temperature and humidity, light intensity, light intensity, soil moisture and $\mathrm{CO}_{2}$ concentration, etc, at the same time in order to create the best environment for the growth of crops[1-3]. With the increasing application of greenhouse is more extensive, the flowers shed species has become increasingly diverse and growing acreage. Because of the combined effects of chemical fertilizer application and other unpredictable factors, more and more diseases are followed, and the fungal disease is the main reason[4]. It's extremely fast, make the other flowers in the greenhouse to the disease, directly affect the production of our country flowers. In order to take effective preventive measures to control the occurrence of diseases, to restore the economic losses caused by the disease, so it is very important to study the disease spot image segmentation for the flower for plant diseases problem.

This paper presents a method based on a Lab color space[5] and Otsu threshold method to segmentation leaves spot of orchid. Lab color space has the characteristics of equally spaced and color difference, which is very suitable for color measurement and comparison in the case of small color difference.

\section{Materials and Methods}

Because the choice of different camera equipment will also make a certain impact on the quality of the collection of pictures, The Lab color space is the most uniform color space, it has nothing to do with equipment, more suitable for close to natural light[6]. In this paper, a component of the Lab space is used to segment the image of the disease leaf of the orchid by adopting the method of Otsu. As shown in figure 1, the segmentation method of leaves disease spot of flower is as follows this paper: (1)The color space of the image of the flower disease image is transformed from the RGB model to the Lab model, and then the a model is extracted from the Lab model; (2) The use of Otsu for image segmentation in the a components, to obtain the binarization image of leaves disease spot of flowers; (3) Using mathematical morphology to smooth filtering and filling of the segmented images, removing the isolated points and improving the quality of the image after segmentation; (4) The binarization image after threshold segmentation just reflect the shape characteristics of the disease spot, but does not show the specific form of color, So you need to reset the R, G, B color channels, retain the original color part of the binarization figure which has split out, restore the color image by cat, finally get the leaf disease spot image.

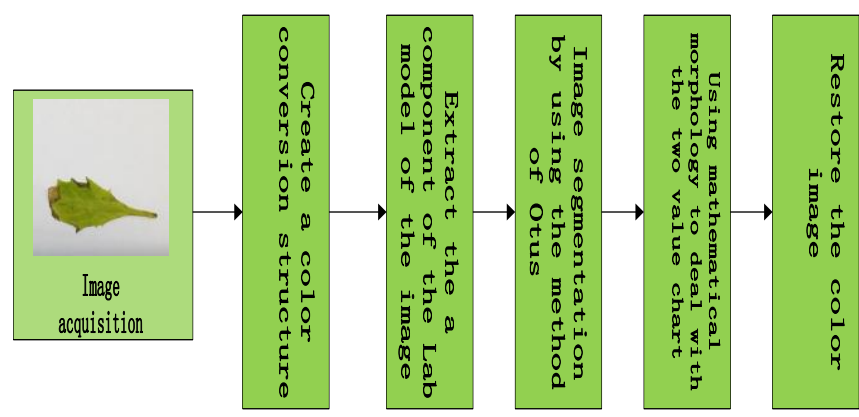

Figure 1: The flow chart of the segmentation algorithm is adopted in this paper

\subsection{Experimental environment and image acquisition}

The hardware of image acquisition and processing system of this study mainly consist of a digital camera, a platform and a computer (Lenovo Y460, clocked at P, 2.40 2G, 500G hard disk memory), MATLAB is used for programming software. Specific operation is to use a digital camera to taking pictures for orchids with diseased spots puting in place of shelves nearly. Using uniform resolution and other settings to a fixed value, to ensure that the acquisition is in the same condition.Finally, the two kinds of orchid leaf images are stored in JPEG or BMP format, as the research object of the experiment. Disease image After acquisition as shown in figure 2 and figure 3 .

\section{Volume 4 Issue 12, December 2015}




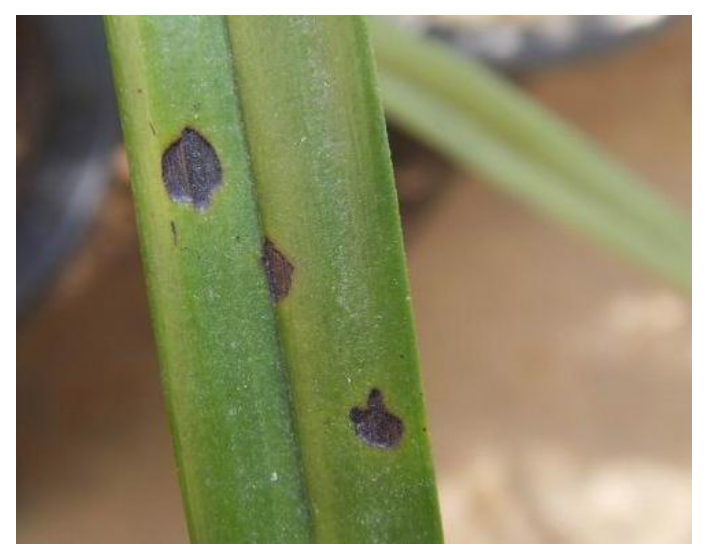

Figure 2: The orchid black rot

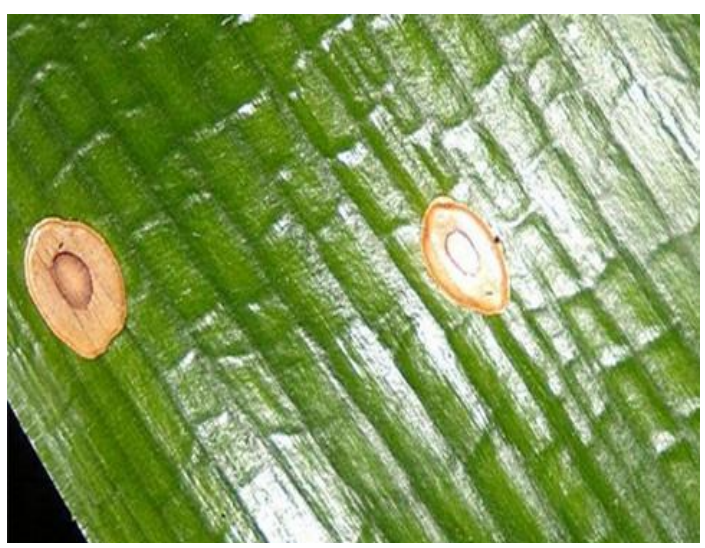

Figure 3: The orchid Anthrax

\subsection{Color Image Preprocessing}

Selecting the color space in color image processing is essential, different color space and background have different application purpose. Generally there is RGB, YUV, HSI and HSV color space model as a choice.The traditional segmentation is generally carried out in the RGB color image, and the selection of this paper is based on the Lab color space. Lab space make a subdivision of brightness and chromaticity index level difference, has the high resolution color, more suitable for the condition of less chromatism for color analysis. Lab color model make up for the deficiency of the RGB color model, it means that the color information which RGB can describe all can be got in Lab space mapping. The Lab color model, L said the brightness, a and b said the two color channel. L can range from 0 to 100 from deep to shallow, a includes color from dark green to grey to light pink, $\mathrm{b}$ from light blue to gray to yellow. As a result, the color after mixed will have a bright effect, then can make the segmentation of disease spot features more obvious. The conversion formula of RGB image into Lab image is:

$$
\left(\begin{array}{l}
X \\
Y \\
Z
\end{array}\right)=\left(\begin{array}{lll}
0.490 & 0.310 & 0.200 \\
0.177 & 0.812 & 0.011 \\
0.000 & 0.010 & 0.990
\end{array}\right)\left(\begin{array}{l}
R \\
G \\
B
\end{array}\right)
$$

$$
f(x)= \begin{cases}7.787 x+0.138 & x \leq 0.008856 \\ \sqrt[3]{x} & x>0.008856\end{cases}
$$

The orchid black spot as an example, R, G, B component of color image is shown in figure4; Convert RGB format to Lab format and then extract the $\mathrm{A}$, the $\mathrm{B}$ component as shown in figure5; We can see that the lesion image features more obvious under Lab space, it can show the difference between the normal part and the disease part, but also can achieve the virtual background, reduce interference. So, this paper chooses a component in Lab space for disease spot image segmentation.
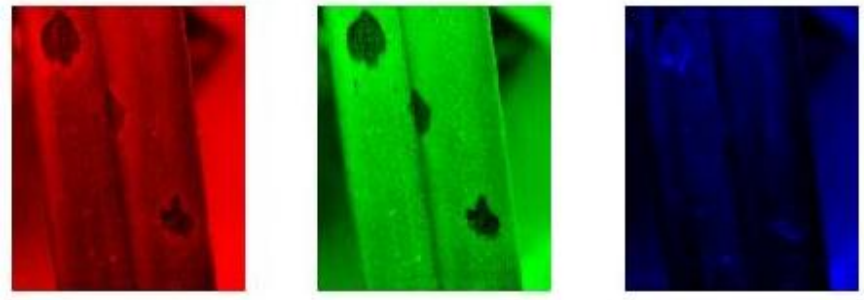

Figure4: RGB model of each component
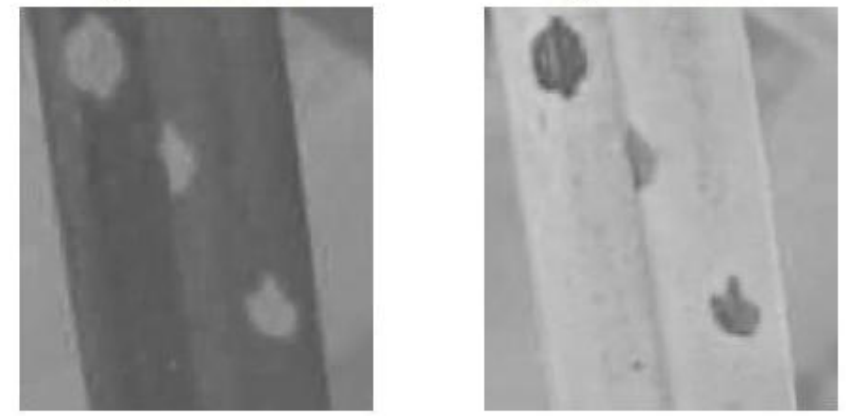

Figure5: Each component of the Lab model

\subsection{Threshold Segmentation}

The traditional image segmentation is mainly can be divided into two kinds:classical image segmentation method and image segmentation method combining the specific theory. In the classical method[7-8]. The segmentation based on threshold and edge detection and the segmentation based on gray level change in the images regional information could be used as a representative. Camera photographed image will be affected by the brightness of the light, the traditional recognition method of vegetable disease spot based on threshold segmentation cannot remove the influence of uneven illumination of image. The other method is to integrate some special subject theory, such as statistics, fuzzy theory, mathematical morphology with image segmentation, so as to improve the accuracy of segmentation to improve the accuracy

\section{Volume 4 Issue 12, December 2015}




\section{International Journal of Science and Research (IJSR) \\ ISSN (Online): 2319-7064}

Index Copernicus Value (2013): 6.14 | Impact Factor (2014): 5.611

of segmentation, but these methods only consider the discrete degree of the color information, without considering space relation of pixels, automatic determination of the threshold is difficult.

Otsu method is derived on the basis of the principle of least squares, the basic idea is to divide the histogram of the image as the image segmentation threshold, then the image is divided into two parts, namely, background and target, and calculate the variance respectively. When the variance between the two parts is bigger, the more greater difference of the two parts, when calculate the variance is the biggest, then this grey value is the threshold of image segmentation. The between-cluster variance threshold segmentation method is simple, fast processing speed, and regarded as the select better way of threshold automatically, Using the maximum variance between the image segmentation, the probability of error segmentation is reduced, if there is an error segmentation, they can make a smaller variance between calculated. The method of Otsu method is simple and fast processing speed, which is regarded as a better method in choosing threshold automatically. When the error segmentation occurs, the variance between the two classes can be reduced.

\subsection{Reduction of Disease Spot Color Images}

The binarization image after threshold segmentation s can intuitively reflect the contour features of disease spot, but does not show the specific form of color, So you need to reset the R, $\mathrm{G}$, B color channels, retain the original color part of the binarization figure which has split out, restore the color image by cat, finally get the leaf disease spot image.

\section{Experimental Results and Analysis}

This article chooses color images of orchid leaf with disease spot on MATLAB7.1 platform for experiments. To deal with the orchid black spot and anthracnose. First to convert images from the RGB color space model to the Lab model, then to grayscale image, The effect of the segmentation by adopting the Otsu method are shown in figure 6 below.

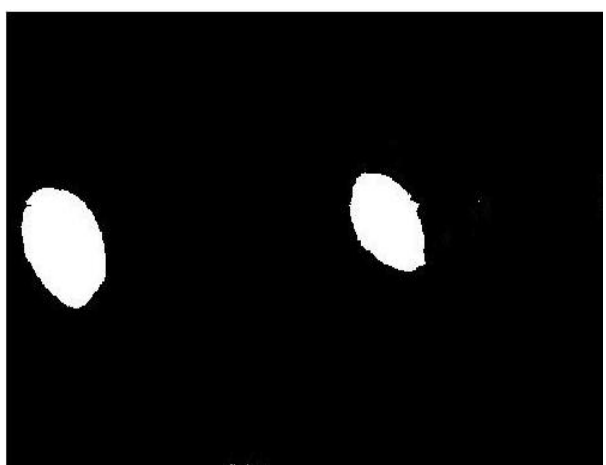

a) The image after segmentation of the orchid black spot

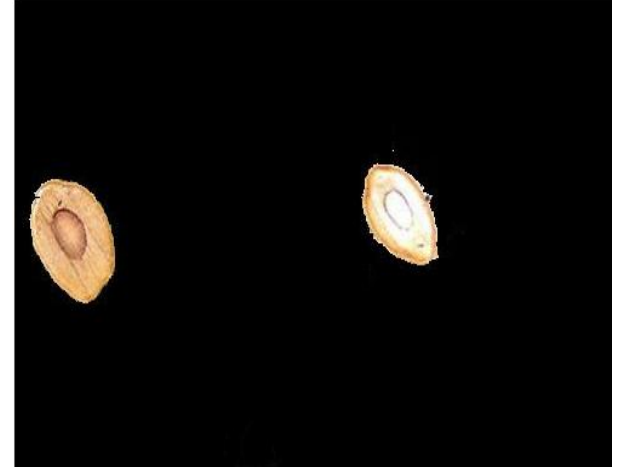

b) Color image of orchid black spot after reduction

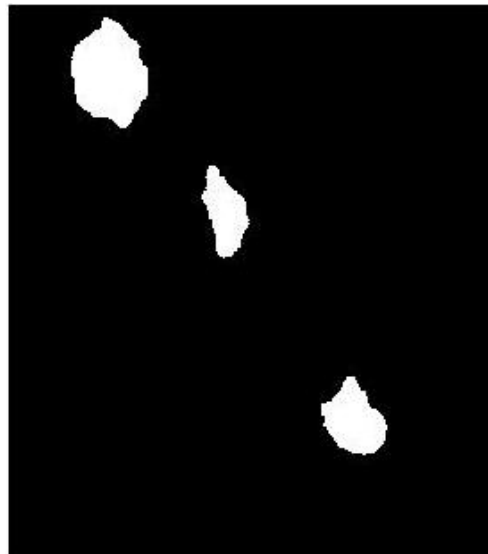

c) The image after segmentation of the orchid Anthrax

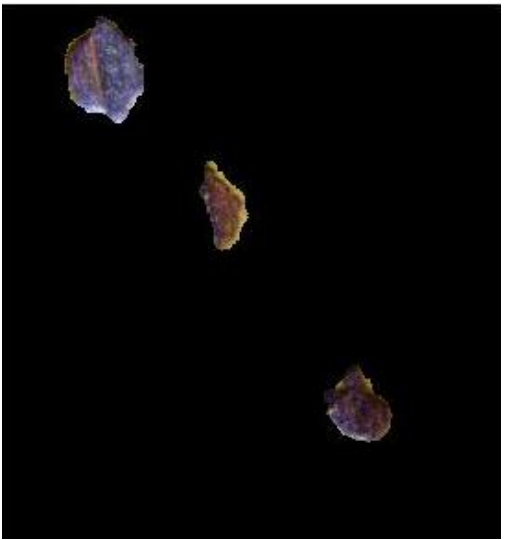

d) Color image of orchid Anthrax after reduction Figure 6: The effect of the segmentation by adopting the Otsu method

\section{Conclusion}

This paper mainly discusses the choice of color space model and introduce the advantages and disadvantages of various kinds of segmentation method for image segmentation. Finally, according to the characteristics of the image, we determine the last should choose what kind of color space, and what kind of image segmentation algorithm, which is the most effective segmentation algorithm. Through experiment, it shows that using Otsu method to segment the orchid leaf disease image on the model of a component in Lab color space, can effectively isolate the disease spot, provide good results for the subsequent feature extraction. 


\section{References}

[1] Hu Xiaohong, Li Bingjun, Xi Lei. Multi-instance graph approach to wheat leaf disease segmentation [J]. Transactions of the Chinese Society of Agricultural Engineering (Transactions of the CSAE), 2012, 28(13): 154 159.

[2] WANG ping, TANG jiang-feng, WANG Bo, WANG Li-di. Image segmentation method of plant leaves based on mathematical morphology [J]. Acta Agriculturae Zhejiangensis, 2012,24(3):509-513.

[3] HUANG Ling, HU Bo, CAO Nai-wen. The Novel Fries-Counting Method Based on Image Processing [J].Hubei Agricultural Sciences, 2012, 51(3):1880 1882

[4] Xun Yi, Bao Guanjun, yang Qinghua, et al. Automatic segmentation of touching corn kernels in digital image [J]. Transaction of the Chinese Society for Agricultural Machinery,2010,41(4):163-167.

[5] Bieniek A, Moga A.An efficient watershed algorithm base on connect components[J]. Pattern Recognition, 2000(3): 907 916.

[6] Wang Kaiyi, Zhang Shuifa, Yang Feng, et al.Online segmentation of clustering diced-potatoes using watershed and improved MRF algorithm [J]. Transaction of the Chinese Society for Agricultural Machinery,2013,44(9):187-192

[7] Xie Zhiyong, Zhang Tiezhong, Zhao Jinying. Ripened strawberry recognition based in Hough transform [J]. Transaction of the Chinese Society for Agricultural Machinery,2007,38(3):106-109.

[8] Xiang Rong, Ying Yibin, Jiang Huanyu, et al. Recognition of Overlapping Tomatoes Based on Edge Curvature Analysis[J].Transaction of the Chinese Society for Agricultural Machinery, 2012,43(3):157-162.

\section{Author Profile}

Danni Ci is reading the M.S. degrees in Mechanical Engineering from Tianjin University of Technology and Education . 